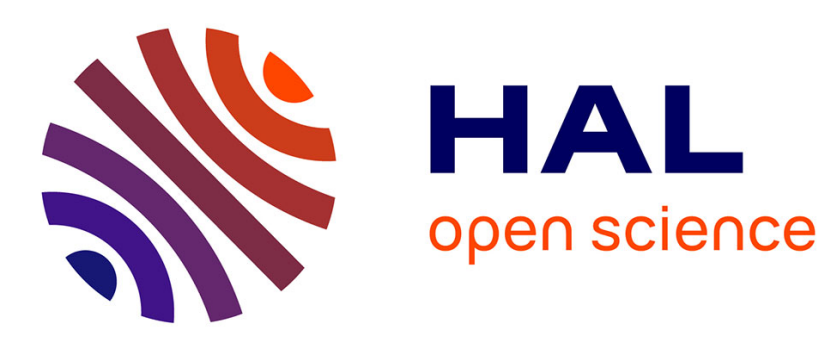

\title{
Essai sur les taillis de chêne vert et de chêne pubescent
} R. Jolain

\section{- To cite this version:}

R. Jolain. Essai sur les taillis de chêne vert et de chêne pubescent. Revue forestière française, 1961, 2, pp.81-89. 10.4267/2042/24345 . hal-03386253

\section{HAL Id: hal-03386253 https://hal.science/hal-03386253}

Submitted on 19 Oct 2021

HAL is a multi-disciplinary open access archive for the deposit and dissemination of scientific research documents, whether they are published or not. The documents may come from teaching and research institutions in France or abroad, or from public or private research centers.
L'archive ouverte pluridisciplinaire HAL, est destinée au dépôt et à la diffusion de documents scientifiques de niveau recherche, publiés ou non, émanant des établissements d'enseignement et de recherche français ou étrangers, des laboratoires publics ou privés. 


\title{
REVUE FORESTIERE FRANÇAISE
}

1961

FÉVRIER

$\mathrm{N}^{\circ} 2$

Dans ce numéro: R. Jolain: Essai sur les taillis de chêne vert et de chêne pubescent. - P. Simonneau et E.-F. DebazaC: Les cyprès des Ajjers $\mathrm{H}$. Gaussen: A propos du cyprès des Ajjers. Son intérêt forestier. - Ph. Hatt: Avenir proposé pour la forêt de la Harth. - M. Cointat: Les friches et les forêts du département de la Haute-Marne.

\section{ESSAI SUR LES TAILLIS DE CHÊNE VERT ET DE CHÊNE PUBESCENT}

\author{
RAPPORT PRÉSENTÉ PAR R. JOLAIN \\ Ingénieur général des Eaux et Forêts
}

La formation des taillis de chêne vert et de chêne pubescent soit en peuplements purs, soit en peuplements mélangés, constitue dans l'ensemble des départements méditerranéens: Drôme - Hérault Gard - Bouches-du-Rhône - Vaucluse - Basses-Alpes - AlpesMaritimes - Aude - Pyrénées-Orientales et Corse une partie importante de la surface boisée.

Celle-ci s'établit pour les seules forèts soumises au Régime forestier à environ 210000 hectares et se répartit dans chacune des conservations intéressées :

\begin{tabular}{|c|c|c|c|c|}
\hline Conservation & $\begin{array}{c}\text { Forêts } \\
\text { domaniales }\end{array}$ & $\begin{array}{c}\text { Forêts } \\
\text { communales }\end{array}$ & Total & Département \\
\hline \multirow[t]{2}{*}{ - } & - & - & - & 一 \\
\hline & (ha) & (ha) & (ha) & \\
\hline $28^{\ominus}$ - Valence ... & 一 & 16074 & 16074 & Drôme \\
\hline $36^{\circ}-$ Montpellier & 2592 & 13790 & 16382 & Hérault \\
\hline $37^{\mathfrak{e}}-$ Nîmes $\ldots \ldots \ldots \ldots$ & 613 & 43848 & 44461 & Gard \\
\hline $38^{e}$ - Aix-en-Provence $\ldots$ & 3783 & 29687 & 33470 & $\begin{array}{c}\text { Bouches-du-Rhône } \\
\text { Vaucluse }\end{array}$ \\
\hline $39^{e}-$ Digne $\ldots \ldots \ldots \ldots$ & 9000 & 14000 & 23000 & Basses-Alpes \\
\hline $40^{e}-$ Nice $\ldots \ldots \ldots \ldots \ldots$ & 4374 & 25835 & 30209 & $\begin{array}{c}\text { Alpes-Maritimes } \\
\text { Var }\end{array}$ \\
\hline $41^{e}-$ Ajaccio........ & 3500 & 24000 & 27507 & Corse \\
\hline \multirow[t]{2}{*}{$35^{\circ}$ - Carcassonne } & 4140 & 7261 & 11401 & $\begin{array}{c}\text { Aude } \\
\text { Pyrénées-Orientales }\end{array}$ \\
\hline & 28002 & 174495 & 202497 & \\
\hline
\end{tabular}


Pour plus de $6 / 7$, ces boisements appartiennent aux Communes.

Cette statistique doit être complétée par l'indication du chiffre des forêts non soumises au Régime forestier: ce chiffre, d'après les données de la statistique Daubrée ressort, pour ces 11 départements à 609000 hectares. Pour être rigoureux, il devrait englober les formations analogues, principalement de chêne pubescent, des autres départements.

On peut néanmoins avancer avec certitude que plus de 800000 hectares, soit approximativement le $15^{\mathrm{e}}$ de la superficie forestière de la France sont couverts par ces formations à taux de productivité très bas et dont une grande partie peut être considérée comme fortement dégradée.

Le régime ancestral du taillis simple, assorti parfois de réserves sur souches ne dépassant pas le stade du baliveau, n'était générateur que de produits de valeur financière peu élevée : bois de feu, charbon de bois, écorces. Mais jusqu'à la première décade du siècle, cette valeur était stabilisée par une économie de subsistance et par une petite industrie artisanale.

Le déclin commence avec l'apparition du pétrole et de ses dérivés, avec celle de l'électricité, avec le développement des transports qui ont transformé, lentement au début, à un rythme de plus en plus rapide ensuite, les conditions de la vie rurale.

Certaines circonstances exceptionnelles - la guerre 1940-45 en redonnant à ces produits une valeur momentanée, n'ont été en fait que des paliers de cette descente continue.

Aujourd'hui, le déclin est total pour l'écorce, presque total pour le charbon de bois et s'aggrave d'année en année pour le bois de feu.

Cette désaffection se traduit par le pourcentage en progression continue des coupes invendues pendant la période 1954-1959: $59 \%$ dans la $28^{\circ}$ Conservation, de 60 à $93 \%$ dans la 36e, de 49 à $77 \%$ dans la $37^{\mathrm{e}}$, de 3 à $60 \%$ dans la $38^{\circ}$, de 31 à $81 \%$ dans la $39^{\circ}$, de 50 à $85 \%$ dans la $40^{\mathrm{e}}$.

Notre Administration ne pouvait rester indifférente à cette situation et à ses répercussions particulièrement sur les Communes propriétaires, communes pauvres pour lesquelles le revenı de leur taillis constituait soit une ressource financière, soit une ressource d'affouage et à laquelle elles restaient attachées.

Il était apparu nécessaire de procéder à une étude aussi précise et complète que possible du problème et de chercher à en dégager les conclusions.

Cette étude s'est étendue aux $28^{\circ}, 36^{\circ}, 37^{\mathrm{e}}, 38^{\mathrm{e}}, 39^{\circ}$ et $40^{\circ}$ Conservations

Elle a donné lieu, le 24 mai 1960, à Aix-en-Provence, à une table ronde à laquelle a été analysée et discutée la documentation recueillie au préalable et dont la synthèse fait l'objet du présent exposé. 


\section{Le Bois}

La production matière principale du taillis de chêne vert et de chêne blanc a toujours été le bois de feu: elle reste aujourd'hui à peu près la seule. Le rendement moyen de l'hectare a fait l'objet de nombreuses expérimentations antérieures, mais celles-ci n'étant pas coordonnées, ne reposant pas sur des bases communes, n'ont qu'une valeur relative.

En l'absence de données précises, on peut néanmoins affirmer que pour un peuplement normalement composé, sans vides, la production dépend de 2 facteurs:

- la nature du sol,

- l'âge d'exploitation.

Le $1^{\text {er }}$ facteur peut être considéré comme constant pour un sol de constitution géologique et de fertilité déterminées dans une forêt non livrée au parcours.

Le $2^{\mathrm{e}}$ est une variable: l'expérience démontre que l'optimum d'exploitation se situe entre 25 et 30 ans.

- Au-dessous, les produits sont de trop faibles dimensions.

- Au-dessus, l'accroissement diminue et le taillis perd rapidement la faculté de rejeter de souche ou de drageonner. Cet âge limite n'a pas été non plus déterminé avec certitude; il dépend directement de la fertilité du sol.

Pour un taillis exploité entre 25 et 30 ans, le rendement oscille entre 20 et 75 stères à l'hectare, en fonction de cette même fertilité.

$\mathrm{Du}$ seul point de vue sylvicole, l'allongement des révolutions est une mesure bénéfique à cause de la remontée biologique qu'elle permet d'espérer.

Du point de vue économique et du point de vue financier, ce n'est qu'une mesure d'attente qui n'influe que peu sur le volume et la valeur des produits.

Compte tenu de ces deux considérants, on peut avancer que la fourchette $25 / 40$ ans correspond le mieux à la fois aux impératifs de conservation du sol, de conservation de l'ensouchement et à la production optima matière et argent.

Il s'agit, même dans les cas les meilleurs, d'un capital travaillant à un taux extrêmement bas, d'une production faible.

Actuellement, la seule utilisation des produits est le chauffage: chauffage des foyers ruraux, pour la plus grande part; chauffage des foyers urbains pour le surplus - encore est-il en régression constante.

Aucune perspective d'utilisation cellulosique n'apparaît jusqu'à présent, et il est peu vraisemblable dans l'état actuel de l'utilisa- 
tion des feuillus pour la production de la cellulose qu'elle se produise dans un avenir appréciable.

La carbonisation des bois est de moins en moins pratiquée et limitée aux besoins de l'exportation et des utilisations spéciales. La production est tombée dans le Var de 12784 T. en 1942 (année exceptionnelle de guerre) à 226 en 1947 et à 69 en 1958.

Une solution pourrait-elle être trouvée dans le choix d'un régime plus approprié?

L'évolution du taillis simple vers le taillis-sous-futaie, qui a fait l'objet de nombreuses expériences, a dû être abandonnée. Le dépérissement rapide des réserves, qu'elles soient issues de rejets de souches ou de drageons, constitue un obstacle cultural; la lenteur de l'accroissement et la faible valeur des produits, qui restent dans la gamme des bois d'industrie, sont un obstacle économique.

Par contre, un furetage progressif permettrait, par réduction périodique du volume de la cépée, d'obtenir des brins plus élevés et plus vigoureux. Mais cette opération assurant un couvert plus complet et plus constant du sol ne saurait être envisagée que du point de vue cultural, et resterait subordonnée aux impératif́s économiques.

\section{Le Pâturage}

Bien que la forêt méditerranéenne n'offre, hors des périodes immédiatement consécutives aux incendies, que des possibilités herbacées limitées, l'économie des formations de chêne vert et de chêne blanc a été longtemps une économie mixte sylvicole et pastorale; la valeur de cette dernière ne le cédant souvent que de peu à la première. Vu sous l'optique de son action sur l'état forestier, le pacage ovin a une influence nocive même dans les peuplements considérés comme défensables et avec charge normale, car il est avéré qu'outre le tassement du sol provoqué par le piétinement, la densité du troupeau, bien que réglementée pour une surface déterminée, n'est pas régulièrement répartie et que la plupart du temps celui-ci pacage constamment les mêmes endroits, ceux qui lui assurent la provende, les vides et les clairières qu'il contribue ainsi à agrandir. Mais l'antagonisme forêt-pâturage apparait aujourd'hui comme périmé avec la diminution des troupeaux locaux: les surfaces défensables n'étant plus que partiellement utilisées. Ainsi, dans l'Hérault, il n'est plus pratiqué que dans 4 forêts communales sur un total de 39; dans le Gard, le nombre des ovins est tombé de 400000 en 1882 à 150000 dont 15 à 20000 cantonnés sur les Causses et ne s'exerce plus que sur les $2 / 3$ des forêts communales.

L'incidence économique se traduit par une diminution des redevances: celles-ci s'établissent entre les chiffres extrêmes de 0,06 NF ( 6 anciens francs) et de 0,40 NF (40 anciens francs) par hectare et par an, avec une moyenne oscillant autour de 0,20 NF 
(20 anciens francs). On peut dire que, malgré les variations sensibles d'une commune ou d'une année à l'autre, le pâturage en fait rapporte peu sinon rien: ni l'adjudication, ni l'ouverture aux troupeaux étrangers ne semblent pouvoir augmenter sensiblement les recettes. Le seul pâturage susceptible de donner un revenu d'une valeur supérieure serait celui exercé dans les cantons non défensables où l'herbe est plus abondante et les rejets peuvent être broutés. On conçoit facilement qu'une telle solution ne soit pas à envisager dans les forêts soumises au Régime forestier.

\section{Autres produits accessoires}

\section{Les menus produits}

Certaines productions spéciales peuvent même se traduire en revenus d'une valeur relativement importante, et supérieure à celle du revenu bois. Le cas le plus typique est celui de la truffe. Dans le Vaucluse, les amodiations de la récolte pour une durée de 5 ans ont été faites, au prix de $3 \mathrm{NF}$ l'hectare. Il faut citer également celui de la bauxite dans une forêt communale du Var bien qu'il s'agisse d'un produit du sous-sol; celle des plantes à huiles essentielles telles que : lavande, thym, sariette.

Mais ce ne sont que des productions accidentelles, ou exceptionnelles.

La levée des écorces ne sera mentionnée que pour mémoire, car elle a pratiquement disparu.

\section{Le revenu financier et la charge de l'impôt foncier}

Cette étude serait incomplète si elle ne faisait pas ressortir:

- le revenu financier,

- la charge fiscale de l'impôt foncier, ramenés à l'hectare de taillis.

La précision dans la recherche du revenu et de l'impôt foncier ne peut être que relative.

Pour délimiter la question, il a été convenu d'étudier dans chacune des Conservations intéressées le cas de 3 forêts ayant une superficie supérieure à 100 hectares peuplées d'un taillis exploité à l'âge de 25-30 ans et donnant un rendement de 50 stères à l'hectare.

Les résultats sont donnés dans le tableau ci-après où les 2 éléments - revenu brut et impôt foncier — sont mis en parallèle, pour mieux illustrer la comparaison. 


\begin{tabular}{|c|c|c|c|c|c|}
\hline \multirow[b]{2}{*}{ Conservation } & \multirow[b]{2}{*}{ Départements } & \multirow{2}{*}{$\begin{array}{c}\text { Nombre } \\
\text { de } \\
\text { Communes } \\
\text { Tests }\end{array}$} & \multicolumn{2}{|c|}{ Pour la décennie 1950-59 } & \multirow{2}{*}{$\begin{array}{c}\text { Observations } \\
\text { Nombre } \\
\text { de coupes } \\
\text { invendues }\end{array}$} \\
\hline & & & $\begin{array}{l}\text { Moyenne du } \\
\text { revenu bois } \\
\text { à l'ha }\end{array}$ & $\begin{array}{l}\text { Moyenne de } \\
\text { l'impôt foncier } \\
\text { à l'ha }\end{array}$ & \\
\hline \multirow[t]{2}{*}{ 一 } & \multirow[t]{2}{*}{ - } & \multirow[t]{2}{*}{ - } & - & - & \multirow[t]{2}{*}{ - } \\
\hline & & & NF & $\mathrm{NF}$ & \\
\hline $28^{\circ}$ & Drôme & 3 & 5,83 & 1,67 & $59 \%$ \\
\hline $36^{\circ}$ & Hérault & 3 & 2,77 & 1,08 & de 60 à $93 \%$ \\
\hline $37^{\bullet}$ & Gard & 3 & 2,84 & 1,33 & de 49 à $77 \%$ \\
\hline \multirow{2}{*}{$38^{\circ}$} & B.-du-Rhône & 6 & 1,46 & 0,80 & \multirow{2}{*}{ de 3 à $60 \%$} \\
\hline & Vaucluse & 10 & 1,57 & 0,94 & \\
\hline 35 & Basses-Alpes & 3 & 3,00 & 0,04 & de 31 à $81 \%$ \\
\hline \multirow{2}{*}{$40^{\circ}$} & Var & 5 & 1,52 & 0,76 & \multirow{2}{*}{ de 50 à $85 \%$} \\
\hline & Alpes-Marit. & 3 & 1,04 & 0,29 & \\
\hline
\end{tabular}

L'analyse de ce tableau fait ressortir:

$1^{\circ}$ la faiblesse extrême du revenu à l'hectare;

$2^{\circ}$ la variabilité d'un département à l'autre de l'impôt foncier et encore plus si on détaillait - de commune à commune ;

$3^{\circ}$ la charge de l'impôt foncier que l'on peut évaluer en moyenne à plus de $40 \%$ du revenu bois brut. Une seule exception, le département des Basses-Alpes où l'équilibre : revenu — impôt - a été maintenu.

Pour être complet, il conviendrait de majorer ce revenu brut des autres sources de revenus dont ceux des menus produits qui, dans certains cas, ne sont pas négligeables et de le minorer des autres charges fiscales (frais de garderie).

La question qui se pose alors à l'esprit est la suivante: comment revaloriser ce capital producteur devant une aussi totale récession?

L'unique solution qui apparaisse valable est la bonification de la forêt par les résineux, ceci malgré l'aggravation du risque d'incendie imputable à cette substitution partielle d'essences.

Le taillis de chêne vert s'enrésine naturellement en Pin d'Alep. La fréquence des feux de forêts, les exploitations dont il est l'objet ont freiné une expansion que favorisent sa rusticité, l'abondance et la remarquable fertilité d'une semence légère à grand rayon de dispersion. 
Il ne s'agit pas là toutefois d'une association véritable et sauf peut-être à la limite de l'aire de l'Alep, sans intervention humaine, le Pin élimine inéluctablement le chêne.

Sans être de qualité, les produits: bois de pâte, poteaux et sciages, surclassent ceux du taillis et trouvent dans les bois d'industrie ou de service une utilisation économique recherchée par une industrie locale à cours de matière première.

La production annuelle d'1 hectare de Pin d'Alep peut être évaluée à $1 \mathrm{~m}^{3} 5 \leftarrow$ sa valeur $=24,00 \mathrm{NF}$.

La production annuelle d'1 hectare de futaie de Pin noir d'Autriche peut être évaluée à $3 \mathrm{~m}^{3} 5-$ sa valeur $=48,00 \mathrm{NF}$.

La production annuelle d' 1 ha de futaie de Pin maritime peut être évaluée à $3 \mathrm{~m}^{3} 5-$ sa valeur $=52,00 \mathrm{NF}$.

La production annuelle d'1 ha de futaie de cèdre, ne peut encore être évaluée avec exactitude.

Les valeurs données peuvent être réduites de moitié si l'on admet que la partie de sol occupée par les résineux n'est que les $50 \%$ de la surface. Elles sont néanmoins fortement excédentaires eu égard à celle du taillis qui ressort à une moyenne de 2,25 NF pour une production annuelle à l'hectare de 1 st. 5 .

$\mathrm{Au}$ Pin d'Alep, peuvent être associés, en fonction de la nature du sol, de la situation littorale ou intérieure des massifs: le Pin maritime, le Pin noir d'Autriche, le Pin Laricio de Corse, le Pin pignon, les Cyprès.

Le taillis de Chêne pubescent se situe dans la zone altitudinale de 5 à 800 mètres. d'où le Pin d'Alep est naturellement éliminé et où il n'est remplacé par aucun autre résineux spontané.

Par contre, les reboisements effectués depuis 80 ans ont démontré l'excellence de la réussite de 2 essences: le Pin noir d'Autriche et le Cèdre, qui constituent, avec la deuxième génération, des forêts pérennes.

Il semble alors qu'une solution s'offre de revaloriser, au moins dans une certaine mesure, ces taillis défavorisés, en substituant à leur production dépréciée, celle d'une gamme d'autres produits plus appréciés. Cette solution est celle de l'enrésinement par voie naturelle ou provoquée en Pin d'Alep, Pin Maritime et Pin Pignon des formations de chêne vert; celle du reboisement par introduction du Pin Noir et du Cèdre des formations de Chêne pubescent.

L'ampleur de la tâche ne doit pas être un obstacle, car il ne saurait s'agir d'une manière générale que d'une œuvre progressivement entreprise et suivie. La constitution de bouquets résineux a fait ses preuves: la dispersion de la graine des semenciers, cèdres principalement et Pin Noir, introduits il y a moins d'un siècle, est une évidence frappante. Celle du Pin d'Alep dans des formations exploitées ou parcourues par le feu ne l'est pas moins. A défaut 
de moyens financiers pris en dehors du F.F.N., parce que la majeure partie des reboisements de ce type ne répond pas aux critères économiques et qu'ils seront toujours sans rapport avec la valeur du capital initial, fonds et superficie, cette méthode vaut d'être étudiée et tentée par petites surfaces dans les forêts des collectivités les plus compréhensives, préparées à cet effet par une vulgarisation adéquate.

\section{III}

\section{Conclusions}

L'étude fait ressortir à la fois l'importance de la superficie des formations de chêne vert et de chêne pubescent et la faiblesse croissante de leur valeur économique.

Elle constate que la seule amélioration susceptible d'être apportée actuellement serait une substitution plus ou moins étendue d'essences.

Mais la réalisation de cette substitution pose un problème de financement.

L’idée vient tout naturellement de s'adresser au F.F.N. Or, celuici est un Fonds de développement économique et son intervention reste subordonnée à la rentabilité ou mieux à la valeur économique du travail considéré.

L'enrésinement des taillis de chêne vert et de chêne pubescent dans leur généralité ne paraît pas devoir entrer dans ces catégories privilégiées. Seule la subvention pourrait être retenue. Toutefois, il s'agit là d'un procédé non négligeable, mais n'agissant que par petites touches et qui ne peut être qu'un palliatif.

A défaut de crédits de tel ordre, on pourrait se tourner vers l'orientation suivante:

Une discrimination devrait être faite entre les deux types de formations :

Les taillis de chêne pubescent, bénéficient de conditions écologiques nettement supérieures à celles des taillis de chêne vert. L'introduction de Pin Noir d'Autriche et du Cèdre y a fait ses preuves: il ne s'agit donc plus d'une expérimentation, mais bien de l'extension d'une méthode reconnue.

Pour la réaliser, la seule solution financière valable serait apportée par l'ouverture de crédits d'investissement attribués aux reboisements n'ayant pas un caractère directement économique. En attendant le résultat espéré des efforts entrepris dans ce sens, il serait imprudent d'ignorer l'effort entrepris par certains départements pour aider les collectivités communales désireuses de procéder à des travaux de reboisement.

Aujourd'hui, dans 1'Hérault, 1000 hectares ont été reboisés de- 
puis 1914 à l'aide de crédits communaux, doublés de crédits alloués par la Commission départementale de reboisement (Flahault).

Le Conseil Général des Alpes-Maritimes a:

- d'une part décidé de porter à $43 \%$ le taux de la subvention qu'elle accordait aux Communes,

- et de prendre en charge $80 \%$ du montant des annuités de remboursement des prêts F.F.N. pour travaux de reboisement.

Le Conseil Général des Basses-Alpes a adopté le principe du remboursement de $66 \%$ de l'annuité des prêts de reboisement aux Communes emprunteuses.

Il faut voir là le commencement d'évolution d'une mentalité jusqu'ici indifférente, pour le moins qu'on puisse dire, aux problèmes forestiers, et qu'il conviendrait de ne pas décourager par une attente dont les motifs, si valables qu'ils puissent être, ne seraient pas compris.

Nous sommes ainsi amenés à préconiser, sous forme de prêts F.F.N., une aide financière, restreinte pour le moment, en raison des circonstances, mais cependant assurée, aux communes - et elles sont peu nombreuses - qui, dans des cas bien définis, même s'ils sont limités, manifesteront une volonté marquée d'améliorer leur patrimoine forestier. Pour le surplus, une action intensive de propagande, qui serait sanctionnée par l'attribution la plus large possible de subventions, devrait être entreprise sur le plan local.

Paris, le $1^{\text {er }}$ décembre 1960.

\section{Les utilisations du Bois dans l'Agriculture}

L'Association pour le Développement des Emplois du Bois, dont le Secrétariat est installé, 10 , av. de St-Mandé à Paris $\left(12^{\mathrm{e}}\right)$, vient de faire paraitre, en collaboration avec la "Revue du Bois ) un numéro spécial qui étudie les nombreuses possibilités d'utilisation du Bois dans l'Agriculture: habitations, hangars et constructions nécessaires à la ferme, etc... 